\title{
Adrenal Hormone Interactions and Metabolism: A Single Sample Multi-Omics Approach
}

\section{(ㅇ)(요 $\Theta$}

Authors

Nicole Bechmann ${ }^{1,2,3,4}{ }^{\circledR}$, Deepika Watts ${ }^{1}$, Charlotte Steenblock ${ }^{2}$, Paal William Wallace ${ }^{1}$, Annette Schürmann ${ }^{3,4}$, Stefan R. Bornstein ${ }^{2}$, Ben Wielockx ${ }^{1}$, Graeme Eisenhofer ${ }^{1,2}$, Mirko Peitzsch ${ }^{1}$

Affiliations

1 Institute of Clinical Chemistry and Laboratory Medicine, Technische Universität Dresden, Dresden, Germany

2 Department of Medicine III, Technische Universität Dresden, Dresden, Germany

3 German Institute of Human Nutrition Potsdam-Rehbruecke, Department of Experimental Diabetology, Nuthetal, Germany

4 German Center for Diabetes Research (DZD), München-Neuherberg, Germany

Key words

adrenal, steroids, catecholamines, TCA-cycle metabolites, preservation of native proteins, cortical-medullary interaction

received 11.01 .2021

accepted after revision $\quad 11.03 .2021$

published online 26.04 .2021

Bibliography

Horm Metab Res 2021; 53: 326-334

DOI 10.1055/a-1440-0278

ISSN 0018-5043

(c) 2021. The Author(s).

This is an open access article published by Thieme under the terms of the Creative Commons Attribution-NonDerivative-NonCommercial-License, permitting copying and reproduction so long as the original work is given appropriate credit. Contents may not be used for commecial purposes, or adapted, remixed, transformed or built upon. (https://creativecommons. org/ licenses/by-nc-nd/4.0/)

Georg Thieme Verlag KG, Rüdigerstraße 14,

70469 Stuttgart, Germany

Correspondence

Dr. Nicole Bechmann

Institute of Clinical Chemistry and Laboratory Medicine, University Hospital Carl Gustav Carus Dresden, Technische Universität Dresden

Fetscherstrasse 74

01307 Dresden

Germany

Tel.: + 49351 45819687, Fax: + 493514587346

nicole.bechmann@uniklinikum-dresden.de
Supplementary material is available under

https://doi.org/10.1055/a-1440-0278.

\section{ABSTRACT}

The adrenal gland is important for many physiological and pathophysiological processes, but studies are often restricted by limited availability of sample material. Improved methods for sample preparation are needed to facilitate analyses of multiple classes of adrenal metabolites and macromolecules in a single sample. A procedure was developed for preparation of chromaffin cells, mouse adrenals, and human chromaffin tumors that allows for multi-omics analyses of different metabolites and preservation of native proteins. To evaluate the new procedure, aliquots of samples were also prepared using conventional procedures. Metabolites were analyzed by liquid-chromatography with mass spectrometry or electrochemical detection. Metabolite contents of chromaffin cells and tissues analyzed with the new procedure were similar or even higher than with conventional methods. Catecholamine contents were comparable between both procedures. The TCA cycle metabolites, cis-aconitate, isocitate, and $\alpha$-ketoglutarate were detected at higher concentrations in cells, while in tumor tissue only isocitrate and potentially fumarate were measured at higher contents. In contrast, in a broad untargeted metabolomics approach, a methanol-based preparation procedure of adrenals led to a 1.3-fold higher number of detected metabolites. The established procedure also allows for simultaneous investigation of adrenal hormones and related enzyme activities as well as proteins within a single sample. This novel multi-omics approach not only minimizes the amount of sample required and overcomes problems associated with tissue heterogeneity, but also provides a more complete picture of adrenal function and intraadrenal interactions than previously possible. 


\section{Introduction}

The adrenal gland integrates neuronal, metabolic, and endocrine signaling molecules central to many physiological and pathophysiological processes. The catecholamines, norepinephrine and epinephrine, are produced in the adrenal medulla and function in fight-or-flight acute stress situations [1,2]. Adrenal-derived steroid hormones, including mineralocorticoids, glucocorticoids, and androgens, are synthesized in distinct zones of the adrenal cortex [3] and fulfill various physiological functions such as regulation of blood pressure, electrolyte homeostasis, and glucose metabolism as well as providing precursors for the synthesis of fully functional sex hormones.

A complex network of autocrine and paracrine pathways regulates steroid and catecholamine biosynthesis/secretion [4]. Bidirectional cross-talk between the adrenal cortex and the medulla is furthermore involved in the regulation of both hormone systems ( $>$ Fig. 1). After neuroendocrine stimulation, adrenocortical cells release steroids, which also diffuse into adjacent chromaffin cells. Binding of steroids to intracellular steroid receptors and subsequent translocation to the nucleus leads to induction of the transcription of specific genes involved in catecholamine production. The glucocorticoid-mediated induction of phenylethanolamine- $N$-methyltransferase (PNMT), the enzyme that converts norepinephrine to epinephrine is well established [5,6]. Conversely, catecholamines regulate steroid biosynthesis in adrenocortical cells in a paracrine manner via binding to $\alpha$ - or $\beta$-adrenergic receptors with resulting transcription of steroidogenic enzymes [7-9].

Pathophysiological conditions can affect this highly regulated system. Pheochromocytomas, primary aldosteronism and hypercortisolism, are associated with an overproduction of adrenal hormones, which can lead to systemic symptoms including endocrine hypertension [10]. In contrast to patients with primary hypertension, patients with pheochromocytomas also exhibit elevated plasma steroid concentrations, demonstrating cortical-medullary interactions [10]. Furthermore, Addison's disease or congenital adrenal hyperplasia $(\mathrm{CAH})$ are respectively associated with insufficient or dysregulated adrenal hormone production, compromising the development of sex characteristics in patients with $\mathrm{CAH}$ [11].

The close interaction between adrenal steroids and catecholamines, and their important role in health and disease, emphasizes the importance of considering both endocrine systems for the development of novel diagnostic and therapeutic approaches. Analytical procedures that allow the simultaneous analysis of different classes of hormones and further metabolites in adrenal-derived tissue is challenging. Furthermore, these methods have only limited informative validity, as they only reflect the present metabolite concentration, but do not provide any information about the affected proteins. Additional procedures must be applied to identify underlying molecular alterations, but those require separate tissue specimens. The use of separate tissue specimens affects the significance of the results, since the ratio of cortex to medulla may differ between different specimens and, in the case of tumor tissue, the heterogeneity of the tumor may affect the results. Moreover, an increased amount of valuable tissue is required, that is usually very limited by the size of the adrenal, especially in animal experiments.

We therefore aimed to develop a sample preparation procedure allowing for a multi-omics approach within a single adrenal-derived sample, either from chromaffin cell culture experiments or from tissue-based sample materials. In addition to the simultaneous analyses of different classes of metabolites, the novel procedure also facilitates the investigation of enzymatic activity and protein expression within the sample by preserving the native protein structure.

\section{Materials and Methods}

\section{Chemicals and reagents}

If not otherwise stated, all reagents were of highest available purity from Sigma Aldrich GmbH (St. Louis, MO, USA) or VWR International (Radnor, PA, USA). Cell culture medium and additives were obtained from Gibco (Thermo Fisher Scientific, Waltham, MA, USA) with the exception of fetal calf serum (Biowest, Riverside, MO, USA).

\section{Model systems and specimens}

Three different model systems were used for development and validation of the new sample preparation procedure: 1 . pheochromocytoma cell lines (Supporting Information); 2. mouse adrenal glands; and 3. pheochromocytoma and paraganglioma tumor specimens.

\section{Mouse adrenal glands}

Mouse adrenal glands were used to establish the multi-omics approach in organ-derived samples. Therefore, C57BL/6J mice (JANVIER, Le Genest-Saint-Isle, France) were bred and housed under specific pathogen-free conditions on a 12-h light/12-h dark cycle. At the age of 8 weeks, the adrenal glands of female mice were removed at $10 \mathrm{AM}$ after sacrificing the mice by cervical dislocation. Adrenal glands were resected, followed by a careful dissection of surrounding fat, and immediately snap-frozen in liquid nitrogen. Samples were stored at $-80^{\circ} \mathrm{C}$ until further processing. Procedures were approved by the Landesdirektion Sachsen.

\section{Tumor specimens}

Twelve tumor tissue samples from surgeries of 11 patients with pheochromocytoma or paraganglioma at the University Hospital Dresden were examined. All patients participated in the Prospective Monoamine-producing Tumor (PMT)-study (https://pmtstudy.pressor.org; ethical approval from the local ethics committee of the Technische Universität Dresden under protocol EK 189062010) and provided written informed consent.

\section{Single sample preparation procedure}

To preserve native protein structure, a special cell disruption buffer (component of Invitrogen Paris Kit, AM 1921, ThermoFisher Scientific) allowing the nuclear and cytoplasmic disruption of cells was used. Cell disruption buffer $(100 \mu l)$ was added to sample tubes placed on ice containing the respective samples of either cells, whole mouse adrenals or 10-15 mg of tumor tissue. Thereby, samples were completely covered with the buffer. After 15 minutes of incubation at $4{ }^{\circ} \mathrm{C}$, samples were homogenized using a tissue grinder (NG010262024, NIPPON Genetics Europe, Düren, Germany). To ensure complete disruption, homogenized samples were again incubated for another 15 minutes on ice. After thorough 


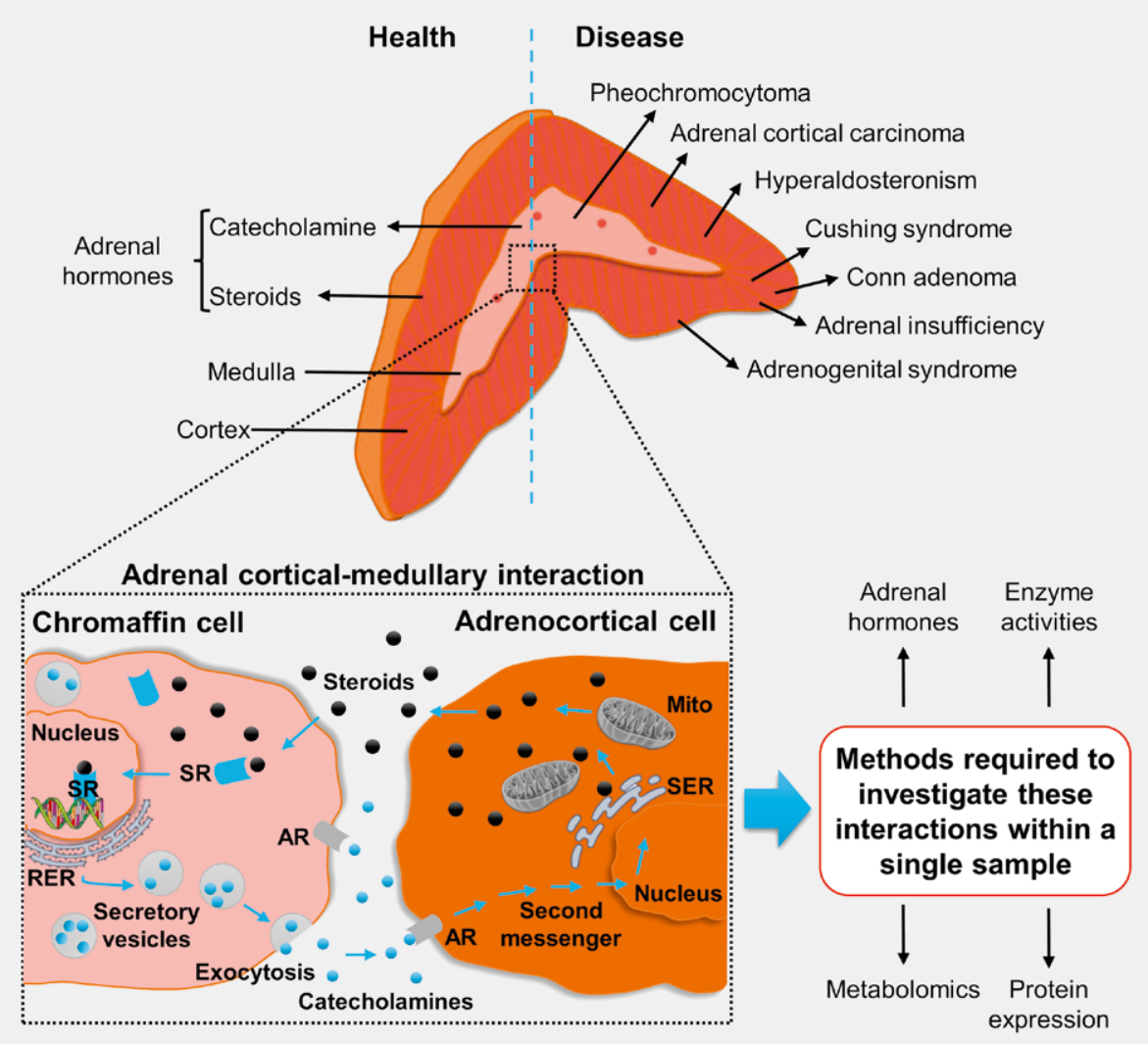

- Fig. 1 Adrenal cortical-medullary interactions in health and disease: The adrenal gland embeds two embryologically and functionally distinct tissues, the steroid producing adrenal cortex and the catecholamine producing adrenal medulla. Neuroendocrine stimulation leads to a release of steroids by adrenocortical cells. Steroids subsequently also diffuse into adjacent chromaffin cells, where they bind to intracellular steroid receptors (SR). Subsequent translocation to the nucleus leads to induction of the transcription of specific genes involved in catecholamine synthesis. Reversely, catecholamines regulate steroid biosynthesis in adrenocortical cells in a paracrine manner by binding to $\alpha$ - or $\beta$-adrenergic receptors (AR) resulting in the transcription of steroidogenic enzymes. Pathophysiological conditions, such as adrenal insufficiency, can affect this highly regulated system. To better understand the complex interaction between the adrenal cortex and the medulla, in particular how they are affected by disease, novel analytical approaches are needed that allow the investigation of different aspects of this interaction in one piece of tissue. SER: Smooth endoplasmic reticulum; RER: Rough endoplasmic reticulum; Mito: Mitochondria.

vortex-mixing for 20 seconds, samples were centrifuged for 5 minutes at $4{ }^{\circ} \mathrm{C}(16000 \times \mathrm{g})$, and supernatants transferred into new sample tubes on ice. For further processing distinct aliquots of lysates were transferred to new sample tubes containing additives required for the respective analyses (Table S1).

\section{Conventional sample preparation procedures}

To investigate the comparability of the newly developed sample preparation procedures with conventional sample preparation protocols, comparable samples were processed with $100 \mu \mathrm{l}$ of commonly used solutions (conventional procedure) as described elsewhere for catecholamines (perchloric acid) [12], TCA-cycle metabolites (cold methanol) [13] and steroid hormones (cold methanol) [14]. For untargeted metabolomics, samples were prepared using $100 \mu \mathrm{l}$ cold methanol. For the conventional sample preparation, samples are also mixed with the corresponding solutions, incubated for 10 minutes and afterwards homogenized using a tissue grinder. After centrifugation of homogenized samples, supernatants were aliquoted similarly to the single sample preparation method using the same additive that was used here for the preparation of the samples (same dilution as for the single sample preparation procedure). For samples derived from cell culture, cell pellets $\left(5 \times 10^{5}\right.$ cells) from the same cell passage prepared on the same days were used for method comparisons (paired samples). Mouse adrenals from 14 individuals were removed as described above. Seven adrenals (mixture left and right adrenal) were prepared using the cell disruption buffer and compared with either the adrenals of the same animal or with the adrenals of the other animals prepared with the conventional procedures (unpaired samples). Separate samples of tumor tissue were prepared for catecholamine, steroid and TCA-cycle metabolites using the conventional sample preparation protocols and compared with an additional sample processed by applying the newly described procedure.

\section{Analytics}

Sample aliquots $(100 \mu l)$ generated with the novel (aqueous lysate plus additive) or the conventional ( $100 \%$ of the additive) sample preparation procedure were utilized for the subsequent analyses. 


\section{Catecholamine measurements}

Norepinephrine, epinephrine and dopamine, and the catecholamine precursor dihydroxyphenylalanine (DOPA) were measured by liquid chromatography with electrochemical detection (LC-ECD) as previously described $[15,16]$.

\section{Steroid hormones and TCA-cycle metabolites}

Adrenal steroid hormones, and TCA-cycle metabolites were determined by LC-MS/MS as described elsewhere $[17,18]$.

\section{Untargeted metabolomics}

For purposes of untargeted metabolomics analysis, an ultra-performance liquid chromatography-ion mobility spectroscopy-high-resolution tandem mass spectrometry (UPLC-IMS-HR-MS/ MS) method was set up on a VION IMS-Quadrupole-Time-of-Flight MS (Waters) coupled to an Acquity i-class UPLC (Waters). For chromatographic separation an XBridge Amide column $(4.6 \times 100 \mathrm{~mm}$, $3.5 \mu \mathrm{m}$; Waters) at $40^{\circ} \mathrm{C}$ was used with mobile phases A [20 mM ammonium acetate in $95 \% / 5 \%(\mathrm{v} / \mathrm{v})$ water/acetonitrile, $\mathrm{pH} 9.1$ ] and $B$ (acetonitrile). Gradient conditions were as follows: 0-10 minutes 15-50\% A, 10-15 minutes 50-95\% A, 15-16 minutes 95-15\% A, 16-23 minutes equilibration with $15 \% \mathrm{~A}$. The mass spectrometer was set to High Definition MSE mode for a mass range of mass to charge ratios $(\mathrm{m} / \mathrm{z})$ from 50 to $1000 \mathrm{Da}$. Capillary voltage was set to $3.0 \mathrm{kV}$, source and desolvation temperatures were 120 and $500{ }^{\circ} \mathrm{C}$, respectively, while cone and desolvation gas flows were set to 50 and $900 \mathrm{l} / \mathrm{h}$, respectively. For fragmentation, collision energy settings were $6 \mathrm{eV}$ (low energy) for $25 \%$ of the scan time before it was ramped from $2856 \mathrm{eV}$ (high energy) for the remaining $75 \%$ of the time. Repeated injections of a leucine-enkephalin solution served as reference for mass corrections.

Resulting data were processed in Progenesis QI (nonlinear), where retention time alignment, peak picking and metabolite identification was performed. Metabolite identification was carried out using the Chemspider plugin searching against the HMDB [19] and KEGG [20] metabolite databases and an in-house metabolite database containing accurate mass, retention times, collision cross section (CCS) values as well as fragmentation patterns derived from standards.

\section{PNMT enzyme activity}

Phenylethanolamine $\mathrm{N}$-methyltransferase enzyme activity determined by the enzymatic conversion of norepinephrine to epinephrine was analyzed with minor modifications by LC-MS/MS as previously described [5]. In brief, to a reaction mixture containing $920 \mu \mathrm{l}$ $0.4 \mathrm{M}$ Tris- $\mathrm{HCl}$ buffer ( $\mathrm{pH} 8.5$ ), $20 \mu \mathrm{l} 2.5 \mathrm{mM} \mathrm{d}_{3}$-S-adenosyl methionine ( $d_{3}$-SAM), $20 \mu \mathrm{l} 2.5 \mathrm{mM}$ norepinephrine, and $40 \mu \mathrm{l}$ tissue/cell lysate were added to a reaction tube, and incubated for 20 minutes at $37^{\circ} \mathrm{C}$. Enzyme activity was quenched by placing samples on ice. For analysis, $20 \mu \mathrm{l} d_{6}$-epinephrine as internal standard $(50 \mathrm{ng} / \mathrm{ml})$, $400 \mu \mathrm{l} 1 \mathrm{M}$ Tris- $\mathrm{HCl}$ ( $\mathrm{pH} 8.6$ with EDTA) and $5 \mathrm{mg}$ dried alumina were added. Then, samples were vortex-mixed for 20 minutes, centrifuged for 30 seconds at $4{ }^{\circ} \mathrm{C}(4500 \times \mathrm{g})$ followed by rejection of the supernatant. The remaining alumina including the bound analytes of interest was washed twice with deionized water, followed by resuspension in water/acetonitrile (98:2, v/v) containing $0.2 \%$ formic acid. The alumina/solvent mixtures were then vortex-mixed for 45 seconds, centrifuged and supernatants, containing the analytes of interest, were finally transferred into respective autosampler vials for analysis.

\section{Protein quantification}

Total protein quantification was performed using the Bradford assay (product number 5000006; Bio-Rad Laboratories, Hercules, CA, USA) according to manufacturer's description.

\section{SDS-PAGE and Western blot analysis}

Further protein analyses for tyrosine hydroxylase (TH), phospho (Serine 40) TH and actin were performed by Western blot analysis. For this, $20 \mu \mathrm{g}$ protein isolated by the single sample preparation procedure (isolated lysate) was mixed with LDS sample buffer and $5 \%$ mercaptoethanol. After denaturation at $99{ }^{\circ} \mathrm{C}$ for 5 minutes, proteins were separated using a $10 \%$, or a $4-12 \%$ gradient SDS-polyacrylamide gel. Proteins were then transferred to a polyvinylidine difluoride membrane by tank blotting. Following the blocking of non-specific binding sites with $5 \%$ skimmed milk powder and $2 \%$ bovine serum albumin in TBS-T at room temperature, membranes were incubated with anti-TH (Novus, NB300-109; 1:1000), anti-phospho (S40) TH (abcam, ab51206; 1:500), and anti-Actin (Millipore, MAB1501R; 1:1000) for 2 hours at room temperature followed by an overnight incubation at $4{ }^{\circ} \mathrm{C}$. The following steps were performed as previously described [21].

\section{Data analysis}

Box-plots were used to present unpaired samples (adrenals), with medians shown as straight lines within the boxes. All statistical analyses consider the numbers ( $\mathrm{n}$ ) of biological and technical replicates within independent experiments. If not otherwise indicated, testing for agreement of different preparation procedures was carried out for paired samples using Wilcoxon signed rank test and for unpaired data Mann-Whitney rank sum test (Shapiro test for normal distribution) performed by SigmaPlot 12.5 (Systat Software $\mathrm{GmbH}$, Erkrath, Germany).

\section{Results}

\section{Single sample preparation procedure for multi-omics studies in adrenal-derived sample materials}

Basis for the novel single sample preparation procedure for multi-omics studies built the identification of a sample disruption procedure, which is suitable for the isolation of native proteins and at the same time allows for extraction of both polar and non-polar compound classes within the same sample. The incubation in a special cell disruption buffer in combination with a mechanical homogenization has been proven to be suitable for digestion of different sample materials while preserving native protein [5]. A five-step sample preparation procedure (Fig. S1) was established to enable multi-omics studies in single adrenal-derived specimens. In order to investigate the suitability of the novel single sample preparation procedure, cellular contents of catecholamines and TCA-cycle metabolites in pheochromocytoma cell lines (MPC and PC12) were analyzed using both conventional and novel preparation procedures (Supporting Information). Dopamine and norepinephrine were detected in similar concentrations in both cell lines, regard- 
less of the preparation method (Fig. S2). For TCA-cycle metabolites, the newly established sample preparation led to similar or even increased concentrations compared to the conventional method (Fig. S2). Differences in metabolite concentration followed the same direction in both cell lines confirming that observed differences are not due to cell line- or matrix-dependent effects. The cell line experiments demonstrated the suitability of the novel procedure for the first time and thus allowed the investigation of more complex tissues.

\section{Multi-omics analysis of mouse adrenal glands}

Adrenals from C57BL/6] mice were prepared with the novel or conventional sample preparation procedures for analyses of catecholamines and steroid hormones. The concentrations of dopamine, norepinephrine and epinephrine were comparable between the two procedures ( $\mathbf{F i g}$. 2a). For steroid hormones, similar concentrations were observed with the single sample preparation procedure with the exception for progesterone, for which a 23-fold higher $(p=0.001)$ adrenal content was analyzed compared to the conventional procedure ( $\mathbf{F i g}$. $\mathbf{2 b}$ ).

In conjunction with the aforementioned targeted analytical approaches to investigate adrenal metabolites, further analyses using an untargeted metabolomics approach was performed. Overall, 7968 analytical features, defined by certain mass spectrometry derived signals based on chromatographic retention time and accurate mass were observed. Forty-one percent were found to be identical in both preparation protocols with another $39 \%$ only observed in samples prepared with pure methanol and the remaining $20 \%$ exclusively seen using the newly established protocol ( $\mathbf{F i g .} \mathbf{2 c}$ ). However, from those features 276 metabolites were identified using an in-house library search as well as the online KEGG database. Thereby, $69 \%$ of identified metabolites were observed after applying both methods and another $30 \%$ exclusively identified in pure methanolic samples ( $\mathbf{F i g}$. 2c). Nevertheless, by applying an online pathway analysis tool using the identified metabolites, similar metabolites and respective pathways were observed for both preparation strategies ( $\triangleright$ Fig. $\mathbf{2 d}$ ).

The described preparation protocol further allows the isolation of native proteins for both immunochemical and functional analyses. PNMT enzyme activity was analyzed in individual adrenal glands (> Fig. 3a) [5]. In addition, a semi-quantitative western blot analysis was performed to determine individual differences between five C57B/6 Jild type mice in the expression of tyrosine hydroxylase (TH) and of the active phosphorylated protein, phosphor (S40) TH, catalyzing the conversion of L-tyrosine to L-DOPA ( $\vee$ Fig. 3b). Proteinbased normalization of analytical data is furthermore available due to quantification of protein contents. This is especially important because of intra-individual asymmetry of the adrenal glands and differences between sexes (data not shown).

\section{Investigation of the metabolism of chromaffin cell tumors}

Aliquots from 12 tumor tissue samples derived from 11 patients with histologically proven pheochromocytoma or paraganglioma a

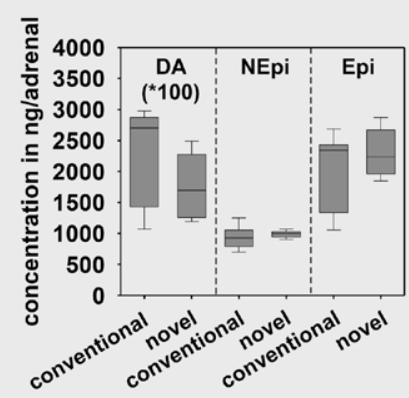

c

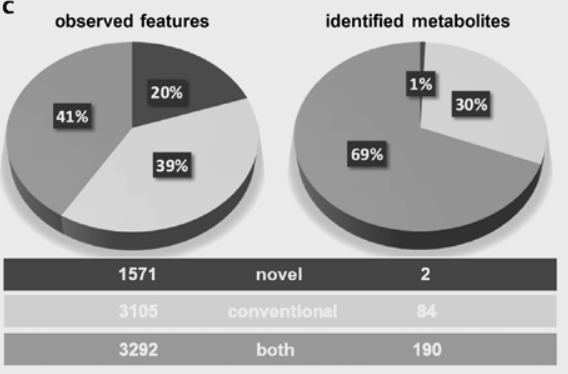

b

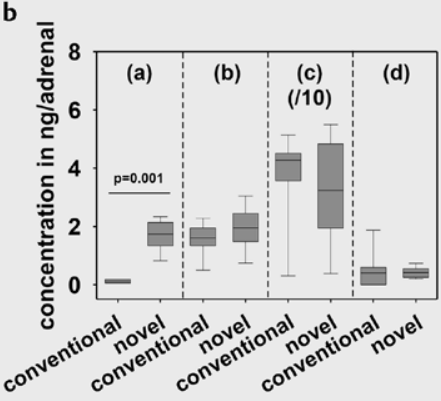

d

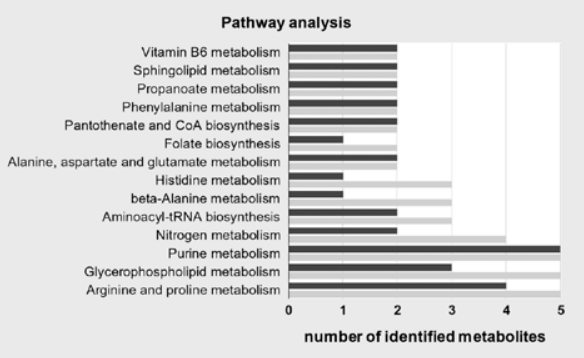

Fig. 2 Multi-omics data observed in adrenals derived from mice using the novel and conventional procedure: a Adrenal tissue contents of catecholamines dopamine (DA), norepinephrine (NEPI) and epinephrine (EPI), and b steroid hormones [(a) progesterone; (b) 11-deoxycorticosterone; (c) corticosterone; (d) aldosterone] covering the two major hormone synthesis pathways of the adrenal. Box-plots showing hormone concentrations analyzed in 5-7 adrenals, medians are given as straight lines within boxes, respectively. Asterisks show significance of differences after Mann-Whitney Rank Sum Test, ${ }^{*}$ p $<0.001$. b Number of observed analytical features and identified metabolites using an untargeted metabolomics approach. c Pathway enrichment analysis performed under www.metaboAnalyst.ca using the identified metabolites in both types of samples. Results for the novel procedure are shown in dark grey, light grey presents results derived from the conventional preparation protocol. 


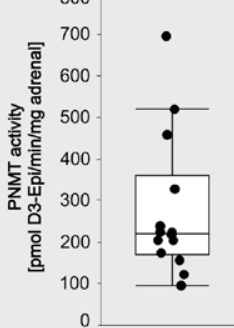

b

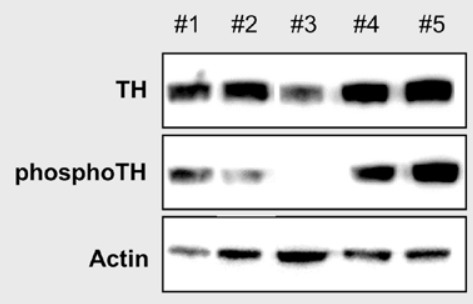

- Fig. 3 Functional and immunochemical analysis of proteins in samples derived from the novel preparation procedure: a PNMT enzyme activity analyzed in 12 individual mouse adrenals. b Western blot analysis of 5 individual mouse adrenal glands (\#1-5) demonstrating the biological variance of tyrosine hydroxylase (TH) and phospho (Serine 40) TH in individual adrenal glands compared to actin as reference protein.

( $>$ Fig. 4a) were prepared two times, either using the novel or the conventional preparation procedure. For dopamine, norepinephrine and epinephrine, no consistent differences in tissue contents were observed between the preparation methods ( $\mathbf{F i g}$. 4b). However, for one tumor (TUM-3), tissue contents of dopamine and norepinephrine were higher using the conventional preparation protocol and, in addition, a 23-fold higher tissue content of epinephrine was analyzed in comparison to the newly established procedure. On the other hand, the analysis of steroid hormone contents in this tumor tissue sample, revealed considerable higher steroid hormone contents, particularly aldosterone $(0.209 \mathrm{ng} / \mathrm{mg}$ tissue), cortisol (4.445 ng/mg tissue) and corticosterone (1.252 ng/mg tissue; - Fig. 4c). In the remaining adrenal pheochromocytomas, tissue cortisol and corticosterone contents varied between 0.150-0.567 $\mathrm{ng} / \mathrm{mg}$ tissue and $0.014-0.084 \mathrm{ng} / \mathrm{mg}$ tissue, respectively, with negligible contents observed for aldosterone and cortisone. Steroid contents measured in extra-adrenal paragangliomas were negligible.

For TCA cycle metabolites measured in tumor tissue samples derived from the two preparation protocols, no differences in tissue contents of citrate, cis-aconitate, $\alpha$-ketoglutarate, succinate ( $\vee$ Fig. $4 d$ ) and malate were observed (Fig. S3). In addition, median values for lactate and 2-hydroxyglutarate, as well as those of the amino acids glutamate, aspartate, glutamine and asparagine, available for both preparation procedures, were similar (Fig. S3). However, the amino acids could not be detected in 8 of 12 samples prepared with the conventional preparation procedure. In contrast, the median concentration of isocitrate was approximately 17 -fold higher $(p<0.001)$ in samples derived from the single sample preparation procedure, combined with non-significant 1.6-fold higher median concentration of fumarate ( $\boldsymbol{F}$ Fig. $\mathbf{4 d}$, and Fig. S3). The latter led to approximately 4.5 to 5.5 lower succinate to fumarate ratios $(p>0.05)$ in tumor samples without and with mutations in a succinate dehydrogenase subunit (SDHx) ( $\triangleright$ Fig. 4d). However, fold differences for succinate to fumarate ratios between tumors with and without SDHx mutations were similar (11.4 vs. 14.1, $\mathrm{p}>0.05$ ) for both preparation protocols.

\section{Discussion and Conclusion}

The present study describes a novel single sample preparation procedure for multi-omics analyses on different experimental and clinical sample materials. In addition to the simultaneous analyses of different metabolite classes, such as adrenal hormones and TCA-cycle and other metabolites, the protocol conserves the native protein for qualitative and quantitative analyses. The main advantage is the availability of complex analytical results from the same sample aliquot. Moreover, the described procedure saves valuable sample material and reduces numbers of required animals. The analyzed sample specimens, pheochromocytoma cell lines, mouse adrenals as well as tumor tissues from patients with pheochromocytomas and paragangliomas (PPGLs) are of particular relevance for studies of the adrenal.

PPGLs exhibit a broad hereditary background caused by more than 20 known PPGL susceptibility genes [22]. These include mutations in genes encoding for $\mathrm{SDHx}$, fumarate hydratase and isocitrate dehydrogenase within the TCA cycle [16]. Screening of TCA-cycle metabolites in PPGL tissue is particularly useful for providing guidance in variant interpretation of genetic testing [16]. This is of importance for patients with mutations in the SDHB subunit, which bears a high risk for metastatic disease, requiring a fast and reliable identification to improve patient outcome [13, 24, 25]. Measurements of succinate to fumarate ratios allow both identification of PPGLs due to SDHx mutations and the quantitative confirmation of the functionality of identified mutations [13]. For this purpose, the comparison of test results derived from both preparation protocols revealed similar differentiation between patients with and without SDHx mutations using tumor tissue succinate to fumarate ratios. Nevertheless, cut-offs for identification of patients with a SDHx mutation established for the conventional procedure [13] require a re-evaluation using the newly established protocol.

The novel procedure will also be of interest to test for other metabolites relevant for tumor biology, such as lactate, which accumulates due to an increased glycolytic energy production in tumor tissue, the so-called Warburg effect. It is known, that tumor lactate levels correlate with increased metastasis, tumor recurrence and poor outcome [23]. Lactate tumor levels were not affected by the sample preparation method (Fig. S4). However, differences for some TCA cycle metabolites in cell culture as well as tumor tissue samples were observed with mostly higher concentrations after applying the novel sample preparation procedure. This may be explained by properties of the cell disruption buffer used. The aqueous buffer has a slightly higher polarity than methanol used for the conventional procedure, which may contribute to the increased recovery of these polar compounds. During the establishment of the new protocol, we also optimized the incubation times, which are longer than in conventional methods. This may also improve cell disruption. However, these differences make it obvious that the concentrations obtained from the two methods are only comparable to a limited extent. Therefore, investigators are requested to decide in favor of the conventional or the herein described procedure, depending on the respective research question and the specific needs.

For PPGL tumor tissue the novel procedure provides several parameters, such as catecholamine contents or the succinate to fumarate ratio within the same sample of tumor tissue. Furthermore, 
a

\begin{tabular}{lcccccc} 
Tumor ID & Legend & Gender & Age & Tumor location & Mutation & Malignant \\
\hline TUM-1 & - & Male & 48 & Adrenal & NF1 & No \\
TUM-2 & - & Male & 51 & Adrenal & NF1 & No \\
TUM-3 & - & Male & 86 & Adrenal & VHL & No \\
TUM-4 & $-\square-$ & Male & 57 & Adrenal & EPAS1 & No \\
TUM-5 & $-\rightarrow-$ & Female & 36 & Extra-adrenal & SDHB & Yes \\
TUM-6 & ---- & Female & 47 & Extra-adrenal & SDHD & Yes \\
TUM-7 & --- & Male & 17 & Extra-adrenal & SDHB & Yes \\
TUM-8a & - & Female & 52 & Extra-adrenal & SDHAF2 & No \\
TUM-8b & - & Female & 54 & Extra-adrenal & SDHAF2 & No \\
TUM-9 & ---- & Female & 52 & Adrenal & HRAS & No \\
TUM-10 & $-\rightarrow-$ & Female & 40 & Adrenal & HRAS & No \\
TUM-11 & $--0-$ & Male & 48 & Extra-adrenal & KIF1 & No
\end{tabular}

b
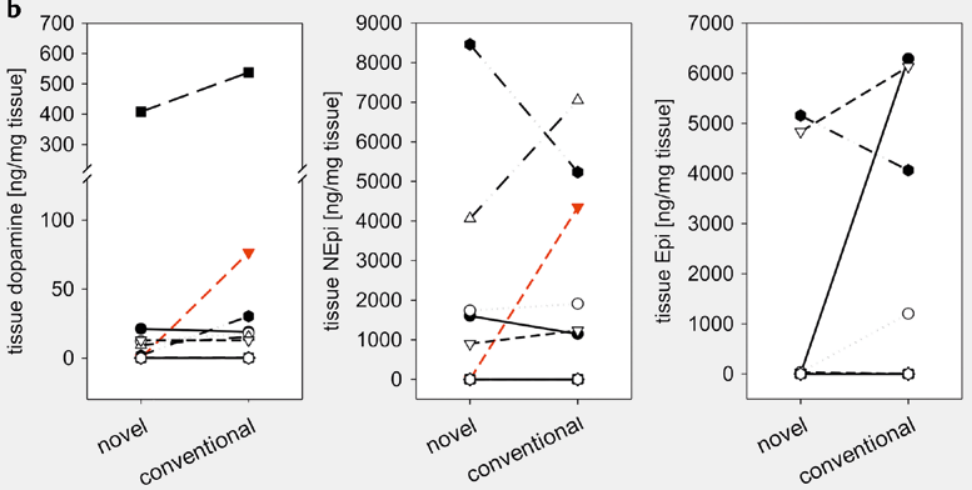

\begin{tabular}{ccccc} 
Aldosterone & $\begin{array}{c}\text { Cortisol } \\
\text { [ng/mg tissue] }\end{array}$ & $\begin{array}{c}\text { Corticosterone } \\
\text { [ng/mg tissue] }\end{array}$ & $\begin{array}{c}\text { Cortisone } \\
\text { [ng/mg tissue] }\end{array}$ \\
\hline TUM-1 & n.d. & 0.567 & 0.016 & n.d. \\
TUM-2 & n.d. & 0.150 & 0.014 & n.d. \\
TUM-3 & 0.209 & 4.445 & 1.252 & 0.089 \\
TUM-4 & n.d. & 0.431 & 0.016 & 0.058 \\
TUM-5 & n.d. & n.d. & n.d. & 0.033 \\
TUM-6 & n.d. & n.d. & n.d. & n.d. \\
TUM-7 & n.d. & n.d. & n.d. & n.d. \\
TUM-8a & n.d. & n.d. & n.d. & n.d. \\
TUM-8b & n.d. & 0.175 & n.d. & n.d. \\
TUM-9 & 0.003 & 0.464 & 0.084 & n.d. \\
TUM-10 & 0.004 & 0.166 & 0.021 & n.d. \\
TUM-11 & n.d. & 0.137 & 0.013 & n.d.
\end{tabular}
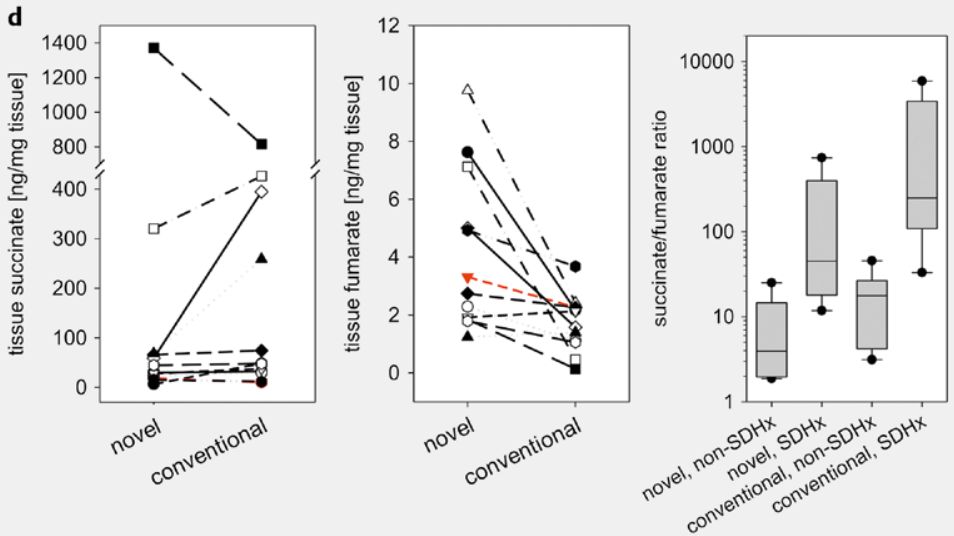

- Fig. 4 Multi-omics approach for simultaneous analyses of catecholamines, steroid hormones and TCA cycle metabolites in 12 PPGL tumor tissue samples derived from 11 patients: a Patient and tumor characteristics. b Tumor tissue catecholamines dopamine, norepinephrine (NEPI) and epinephrine (EPI) analyzed in samples using the novel or the conventional preparation procedure. $\mathbf{c}$ Tissue contents of steroid hormones analyzed in PPGL tumor tissue. $\mathbf{d}$ Succinate and fumarate levels analyzed in PPGL tumor tissue and respective succinate to fumarate ratios used for identification of PPGL patients with SDHx mutation. Box plots showing succinate/fumarate ratio analyzed in 12 different tumors with or without known SDHB mutation, medians are given as straight lines within boxes, respectively. 
this procedure minimizes in particular the impact of tumor heterogeneity on parameter readouts, and allows the identification of contaminations with normal tissue, that improves the quality of the analytical information in tumor related research. In the specific case of TUM-3 ( $\triangleright$ Fig. 4), the novel procedure revealed adrenocortical tissue contamination of the tissue specimen as reflected by high contents of steroid hormones. In contrast, concentrations of dopamine and norepinephrine, but also lactate (Fig. S4) were higher in the tumor specimen prepared with the conventional procedure compared to results observed with the novel procedure. Although tumor heterogeneity is expected, such extensive changes in metabolite concentrations were not observed in any other tissue sample.

The presented method is suitable for quantifying a wide range of metabolites that can be assessed in single patient-derived samples and chromaffin cell culture systems. Catecholamines are an important biomarker for the prognosis of patients with PPGLs [26], and also synthesis and storage of catecholamines in cell model systems are essential features in characterizing cells of chromaffin origin. Moreover, analysis of the two major hormone synthesis pathways of the adrenal is now available within a single adrenal gland. The novel procedure has a significant advantage over the conventional procedure as it allows drawing of a complete picture of the hormonal and metabolic pathways in conjunction with further qualitative but also quantitative information on respective proteins, such as the PNMT enzyme activity.

For the untargeted metabolomics approach, application of the described procedure resulted in smaller numbers of analytical features. Still $41 \%$ of the total number of features were the same in samples from both procedures with another $20 \%$ exclusively observed after application of the novel procedure. This illustrates very well the dilemma of any sample preparation protocol, which always provides a compromise on recoveries of different classes of compounds [27, 28]. In addition, the analytical readout available particularly in untargeted metabolomics approaches strongly depends on settings given in the assay.

In our study, the combination of an aqueous cell disruption followed by the addition of an acid or methanol demonstrated comparable or even improved metabolite concentrations in different sample types and on different polar, less polar and even non-polar substances of interest. In addition, the novel method allows the simultaneous qualification and quantification of proteins, all from a single cell or tissue sample. The new procedure is particularly important for more complex experimental settings or studies with limited sample material. Especially in case of animal experiments, the novel procedure helps to minimize required numbers of laboratory animals. Moreover, it is also expected that the procedure can be applied to cell- and tissue-samples from other origin and an extension of the analyzed metabolite classes is also possible.

\section{Funding}

Deutsche Forschungsgemeinschaft (DFG) CRC/Transregio 205/1 (Project No. 314061271), INST 515/28-1 FUGG and German Ministry of Education and Research and the Brandenburg State 82DZD00302.

\section{Acknowledgements}

The excellent technical assistance of Denise Kaden, Daniela Stanke, Isabel Poser, and Catleen Conrad is greatly acknowledged.

\section{Conflict of Interest}

The authors declare that they have no conflict of interest.

\section{References}

[1] Goldstein DS. Adrenal responses to stress. Cell Mol Neurobiol 2010; 30: $1433-1440$

[2] Berger I, Werdermann M, Bornstein SR et al. The adrenal gland in stress-Adaptation on a cellular level. J Steroid Biochem Mol Biol 2019; 190: 198-206

[3] Miller WL, Auchus RJ. The molecular biology, biochemistry, and physiology of human steroidogenesis and its disorders. Endocr Rev 2011; 32: 81-151

[4] Bornstein SR, Berger I, Scriba L et al. Adrenal cortex-medulla interactions in adaptation to stress and disease. Curr Opin Endocr Metab Res 2019; 8: 9-14

[5] Qin N, Peitzsch M, Menschikowski M et al. Double stable isotope ultra performance liquid chromatographic-tandem mass spectrometric quantification of tissue content and activity of phenylethanolamine $\mathrm{N}$-methyltransferase, the crucial enzyme responsible for synthesis of epinephrine. Anal Bioanal Chem 2013; 405: 1713-1719

[6] Wurtman R], Axelrod J. Adrenaline synthesis: control by the pituitary gland and adrenal glucocorticoids. Science 1965; 150: 1464-1465

[7] Ehrhart-Bornstein M, Bornstein SR, Güse-Behling $\mathrm{H}$ et al. Sympathoadrenal regulation of adrenal androstenedione release. Neuroendocrinol 1994; 59: 406-412

[8] Ehrhart-Bornstein M, Hilbers U. Neuroendocrine properties of adrenocortical cells. Horm Metab Res 1998; 30: 436-439

[9] Lefebvre H, Prevost G, Louiset E. Autocrine/paracrine regulatory mechanisms in adrenocortical neoplasms responsible for primary adrenal hypercorticism. Eur J Endocrinol 2013; 169: R115-R138

[10] Constantinescu G, Langton K, Conrad C et al. Glucocorticoid excess in patients with pheochromocytoma compared with paraganglioma and other forms of hypertension. J Clin Endocrinol Metab 2020; 105 : e3374-e3383

[11] Oelkers W. Adrenal insufficiency. N Engl J Med 1996; 335: 1206-1212

[12] Eisenhofer G, Lenders JW, Goldstein DS et al. Pheochromocytoma catecholamine phenotypes and prediction of tumor size and location by use of plasma free metanephrines. Clin Chem 2005; 51: 735-744

[13] Richter S, Peitzsch M, Rapizzi E et al. Krebs cycle metabolite profiling for identification and stratification of pheochromocytomas/ paragangliomas due to succinate dehydrogenase deficiency. J Clin Endocrinol Metab 2014; 99: 3903-3911

[14] Surowiec I, Koc M, Antti $\mathrm{H}$ et al. LC-MS/MS profiling for detection of endogenous steroids and prostaglandins in tissue samples. J Sep Sci 2011; 34: 2650-2658

[15] Eisenhofer G, Goldstein DS, Stull R et al. Simultaneous liquid-chromatographic determination of 3,4-dihydroxyphenylglycol, catecholamines, and 3,4-dihydroxyphenylalanine in plasma, and their responses to inhibition of monoamine oxidase. Clin Chem 1986; 32: 2030-2033

[16] Bechmann N, Poser I, Seifert $V$ et al. Impact of extrinsic and intrinsic hypoxia on catecholamine biosynthesis in absence or presence of HIF2 $\alpha$ in pheochromocytoma cells. Cancers 2019; 11: 594 
[17] Peitzsch M, Dekkers T, Haase M et al. An LC-MS/MS method for steroid profiling during adrenal venous sampling for investigation of primary aldosteronism. J Steroid Biochem Mol Biol 2015; 145: 75-84

[18] Richter S, Gieldon L, Pang Y et al. Metabolome-guided genomics to identify pathogenic variants in isocitrate dehydrogenase, fumarate hydratase, and succinate dehydrogenase genes in pheochromocytoma and paraganglioma. Genet Med 2019; 21: 705-717

[19] Wishart DS, Feunang YD, Marcu A et al. HMDB 4.0: the human metabolome database for 2018. Nucleic Acids Res 2017; 46(D1): D608-D617

[20] Kanehisa M, Goto S. KEGG: kyoto encyclopedia of genes and genomes. Nucleic Acids Res 2000; 28: 27-30

[21] Bechmann N, Ehrlich H, Eisenhofer $\mathrm{G}$ et al. Anti-tumorigenic and anti-metastatic activity of the sponge-derived marine drugs aeroplysinin-1 and isofistularin-3 against pheochromocytoma in vitro. Mar Drugs 2018; 16: 172

[22] Fishbein L, Leshchiner I, Walter $V$ et al. Comprehensive molecular characterization of pheochromocytoma and paraganglioma. Cancer Cell 2017; 31: 181-193. doi:10.1016/j.ccell.2017.01.001
[23] Doherty JR, Cleveland JL. Targeting lactate metabolism for cancer therapeutics. J Clin Invest 2013; 123: 3685-3692

[24] Eisenhofer G, Lenders JW, Siegert G et al. Plasma methoxytyramine: a novel biomarker of metastatic pheochromocytoma and paraganglioma in relation to established risk factors of tumour size, location and SDHB mutation status. Eur J Cancer 2012; 48: 1739-1749

[25] Bechmann N, Moskopp ML, Ullrich M et al. HIF2 $\alpha$ supports pro-metastatic behavior in pheochromocytomas/paragangliomas. Endocr Relat Cancer 2020; 27: 625-640

[26] Eisenhofer G, Pacak K, Huynh T-T et al. Catecholamine metabolomic and secretory phenotypes in phaeochromocytoma. Endocr Relat Cancer 2011; 18: 97-111

[27] Bi H, Krausz KW, Manna SK et al. Optimization of harvesting, extraction, and analytical protocols for UPLC-ESI-MS-based metabolomic analysis of adherent mammalian cancer cells. Anal Bioanal Chem 2013; 405: 5279-5289

[28] Dietmair S, Timmins NE, Gray PP et al. Towards quantitative metabolomics of mammalian cells: development of a metabolite extraction protocol. Anal Biochem 2010; 404: 155-164 